\title{
ON THE INTITIAL VALUE PROBLEM FOR A PARTIAL DIFFERENTIAL EQUATION WITH OPERATOR COEFFICIENTS
}

\author{
MAHMOUD M. EL-BORAI \\ Department of Mathematics \\ King Abdulaziz University \\ P.0. Box 1540 Jeddah \\ Saudi Arabia
}

(Received May 15, 1979)

ABSTRACT. In the present work it is studied the initial value problem for an equation of the form

$$
L \frac{\partial^{k} u}{\partial t^{k}}=\sum_{j=1}^{k} L_{j} \frac{\partial^{k-j} u}{\partial t^{k-j}} \text {, }
$$

where $L$ is an elliptic partial differential operator and $\left(L_{j}: j=1, \ldots, k\right)$ is a family of partial differential operators with bounded operator coefficients in a suitable function space. It is found a suitable formula for solution. The correct formulation of the Cauchy problem for this equation is also studied.

KEY WORDS AND PHRASES. Partial Differential Equations, Elliptic Operators, Cauchy Problems and General Solutions. 
1. INTRODUCTION.

Consider the equation

$$
\sum_{|q|=2 m} a_{q}(t) D^{q} D_{t}^{k} u=\sum_{j=1}^{k} \sum_{|q|=2 m} A_{q, j}(t) D^{q} D_{t}^{k-j} u,
$$

where $q=\left(q_{1}, \ldots, q_{n}\right)$ is an $n$-tuple of nonnegative integers, and

$$
D^{q}=\frac{\partial|q|}{\partial x_{1}^{q} \ldots \partial x_{n}^{q}}
$$

in which $|q|=q_{1}+\ldots+q_{n} ; D_{t}=\frac{\partial}{\partial t}$, and $m, k$ are positive integers.

It is assumed in equation (1.1) that the following conditions are satisfied;

(a) The coefficients $\left(a_{q}(t),|q|=2 m\right)$ are continuous functions of $t$ in $[0,1]$.

(b) For each $t \in[0,1], \sum_{|q|=2 m} a_{q}(t) D^{q}$ is an elliptic operator.

(c) The coefficients $\left(A_{q, j}(t),|q|=2 m, j=1, \ldots, k\right)$ for each $t \in[0,1]$ are linear bounded operators from $L_{2}\left(E_{n}\right)$ into itself, where $L_{2}\left(E_{n}\right)$ is the set of all square integrable functions on the $n$-dimensional Euclidean space $E_{n}$. (d) The operators $\left(A_{q, j}(t),|q|=2 m, j=1, \ldots, k\right)$, are strongly continuous in $t \in[0,1]$.

In section 2, we shall find a solution $u(x, t)$ of equation (1.1) in a suitable function space so that $t \in(0,1), x \in E_{n}$, and the solution $u(x, t)$ satisfies the following initial conditions

$$
\left.D_{t}^{j} u(x, t)\right|_{t=0}=f_{j}(x), j=0,1,2, \ldots, k-1 .
$$

The uniqueness of the solution of the problem (1.1), (1.2) is also proved. Under suitable conditions $([3],[4])$ we establish the correct formulation of the Cauchy problem (1.1) and (1.2). 


\section{A GENERAL FORMULA FOR THE SOLUTION}

Let $W^{m}\left(E_{n}\right)$ be the space of all functions $f \in L_{2}\left(E_{n}\right)$ such that the distributional derivatives $D^{q}$ with $|q| \leq m$ all belong to $L_{2}\left(E_{n}\right),[8]$.

We shall say that $u$ is a solution of equation $(1.1)$ in the space $w^{2 m}\left(E_{n}\right)$, if for every $t \in(0,1)$ the derivatives $D_{t}^{j} u, j=0,1,2, \ldots, k$ exist and are members of $W^{2 m}\left(E_{n}\right)$ and if $u$ satisfies equation (1.1).

We are now able to prove the following theorem.

THEOREM 1. If $f_{j} \in w^{2 m}\left(E_{n}\right), j=0,1, \ldots, k-1$ and if $4 m>n$, then there exists a unique solution $u$ of the initial value problem (1.1), (1.2) in the space $W^{2 m}\left(E_{n}\right)$.

PROOF. As in $[6]$ the differential operators $\left(D^{q},|q|=2 m\right)$ can be transformed into

$$
D^{q} f=R^{q} \nabla^{2 m} f, f \in W^{2 m}\left(E_{n}\right) \text {, }
$$

where $\nabla^{2}=D_{1}^{2}+\ldots+D_{n}^{2}, R^{q}=R_{1}^{q_{1}} \cdots R_{n}^{q_{n}}, R_{j}$ is the Riesz-transform defined by

$$
R_{j} f=-i \pi^{-\frac{(n+1)}{2}} \Gamma\left(\frac{n+1}{2}\right) \int_{E_{n}} \frac{x_{j}-y_{j}}{|x-y|^{n+1}} f(y) d y,
$$

I is the gamma function, $1=\sqrt{-1}$ and $|x|^{2}=x_{1}^{2}+\ldots+x_{n}^{2}$, (see [1]).

Using (2.1) we see that equation (1.1) is formally equivalent to,

$$
\sum_{|q|=2 m} a_{q}(t) R^{q} \nabla^{2 m} D_{t}^{k} u=\sum_{j=1}^{k} \sum_{|q|=2 m} A_{q, j}(t) R^{q} \nabla^{2 m_{D} k-j} u,
$$

Using the notations

$$
\begin{gathered}
\nabla^{2 m} u=v, \nabla^{2 m} f_{j}=g_{j}, \\
\sum_{|q|=2 m} a_{q}(t) R^{q}=H_{0}(t), \sum_{|q|=2 m} A_{q, j}(t) R^{q}=H_{j}(t),
\end{gathered}
$$

We obtain from (2.2) in a formal way the equation 


$$
H_{0}(t) D_{t}^{k} v=\sum_{j=1}^{k} H_{j}(t) D_{t}^{k-j} v .
$$

Since the operator $\sum_{|\mathrm{q}|=2 m} a_{q}(t) D^{q}$ is elliptic, it follows that the operator $H_{0}(t)$ has a unique bounded inverse $H_{0}^{-1}(t)$ from $L_{2}\left(E_{n}\right)$ into itself, for each $t \in[0,1]$. Applying $H_{0}^{-1}(t)$ to both sides of (2.3) we get

$$
D_{t}^{k} v=\sum_{j=1}^{k} H_{0}^{-1}(t) H_{j}(t) D_{t}^{k-j} v .
$$

Since the operators $R_{j}, j=1, \ldots, n$ are bounded in $L_{2}\left(E_{n}\right)$, it can be easily proved that $H_{j}(t), j=1, \ldots, k$ are bounded operators in $L_{2}\left(E_{n}\right)$ for each $t \in[0,1]$. It is convenient to introduce the following notations in order to complete the proof by considering the problem in a Banach space to be defined below.

Let $A(t)$ denote the square matrix,

$$
A(t)=\left[\begin{array}{ccccc}
\mathrm{H}_{1}^{*}(t) & \mathrm{H}_{2}^{*}(t) & \ldots & \mathrm{H}_{k-1}^{*}(t) & \mathrm{H}_{k}^{*}(t) \\
\mathrm{I} & 0 & \cdots & 0 & 0 \\
0 & \mathrm{I} & \cdots & 0 & 0 \\
\vdots & \vdots & & \vdots & \vdots \\
0 & 0 & \cdots & \mathrm{I} & 0
\end{array}\right] \text {, }
$$

where $H_{j}^{*}(t)=H_{0}^{-1}(t) H_{j}(t), j=1,2, \ldots, k$ and $I$ denotes the identity operator.

Equation $\left(2^{\circ} .4\right)$ can be written in the form

$$
\frac{d V(t)}{d t}=A(t) \quad V(t)
$$

where $\mathrm{V}$ is the column matrix 
and $v_{j}=\quad D_{t}^{k-j} \quad \nabla^{2 m} u$.

$$
\mathrm{v}=\left[\begin{array}{c}
\mathrm{v}_{1} \\
\mathrm{v}_{2} \\
\cdot \\
\cdot \\
\mathrm{v}_{\mathrm{k}}
\end{array}\right]
$$

The column vector $V$ satisfies formally the initial condition

$$
V(0)=\left[\begin{array}{l}
g_{k-1} \\
g_{k-2} \\
\cdot \\
\cdot \\
\cdot \\
g_{0}
\end{array}\right]=G \text {. }
$$

Let $B$ denote the space of column vectors $V$, with the norm

$$
\|v\|=\sum_{j=1}^{k}\left\|v_{j}\right\| L_{2}\left(E_{n}\right),
$$

where $\|f\|^{2} L_{2}\left(E_{n}\right)=\int_{E_{n}} f^{2}(x) d x$.

It is clear that $B$ is a Banach space and $A(t)$ is a linear bounded operator from $B$ into itself for each $t \in[0,1]$. According to the conditions imposed on the coefficients $a_{q}(t), A_{q, j}(t)$, it can be seen that $A(t)$ is strictly continuous on $[0,1]$.

Since $g_{j} \in L_{2}\left(E_{n}\right), j=0,1, \ldots, k-1$, we find that the column vector G is an element of the space B. The abstract Cauchy problem (2.5), (2.6) can be solved by applying the above argument [7]. In other words, there exists for each $t \in(0,1)$ a unique operator $Q(t)$ bounded in the Banach space B such that the formula

$$
V(t)=Q(t) G,
$$


represents the unique solution of the problem $(2.5),(2.6)$ in the space $B$. The operator $Q(t)$ can be represented in the matrix form

$$
Q(t)=\left[\begin{array}{cccc}
Q_{11}(t) & Q_{12}(t) & \cdots & Q_{1 k}(t) \\
Q_{21}(t) & Q_{22}(t) & \cdots & Q_{2 k}(t) \\
\vdots & \vdots & & \vdots \\
Q_{k 1}(t) & Q_{k 2}(t) & \cdots & Q_{k k}(t)
\end{array}\right],
$$

where $\left(Q_{r s}(t), r=1, \ldots, k, s=1, \ldots, k\right)$ are bounded operatros in the space $L_{2}\left(E_{n}\right)$ for each $t$ in $[0,1]$.

Using (2.7) and (2.8) one gets

$$
\begin{aligned}
v_{r}(x, t) & =D_{t}^{k-r} \nabla^{2 m} u(x, t) \\
& =\sum_{s=1}^{k} Q_{r s}(t) g_{k-s}=\sum_{s=1}^{k} Q_{r s}(t) \nabla^{2 m} f_{k-s}
\end{aligned}
$$

From (2.9) we get immediately

$$
u(x, t)=\left(\nabla^{2 m}\right)^{-1} \sum_{s=1}^{k} Q_{k s}(t) \nabla^{2 m} f_{k-s},
$$

where $\left(\nabla^{2 m}\right)^{-1}$ is a closed operator, defined on $L_{2}\left(E_{n}\right)$ and representing the inverse of $\nabla^{2 m}$.

We prove now that the formula (2.10) which we have obtained in a formal way is in fact the required solution of the problem $(1.1),(1.2)$ in the space $w^{2 m}\left(E_{n}\right)$.

Since $\left(\nabla^{2 m}\right)^{-1}$ is a closed operator from $L_{2}\left(E_{n}\right)$ onto $w^{2 m}\left(E_{n}\right)$, it follows immediately from (2.10) that

$$
u \in w^{2 m}\left(E_{n}\right) \text {, }
$$

for each $t \in[0,1]$.

Now the differential operator $\frac{d}{d t}$ in equation (2.5) denotes the abstract derivative with respect to $t$ in the space $L_{2}\left(E_{n}\right)$, i.e. If $f_{t} \in L_{2}\left(E_{n}\right)$ for 
each $t \in(0,1)$, then $\frac{d}{d t} f_{t}$ is defined by

$\lim _{t \rightarrow 0}\left\|\frac{\Delta f_{t}}{\Delta t}-f_{t}^{*}\right\| L_{t} L_{2}\left(E_{n}\right)=0$,

and $\Delta f_{t}=f_{t+\Delta t}-f_{t}$.

Since $\frac{d}{d t}\left(\nabla^{2 m}\right)^{-1} f_{t}=\left(\nabla^{2 m}\right)^{-1} \frac{d}{d t} f_{t}, f_{t} \in L_{2}\left(E_{n}\right)$,

it follows from (2.9) and (2.10) that

$$
\begin{aligned}
\frac{d^{k-r}}{d t^{k-r}} u & =\left(\nabla^{2 m}\right)^{-1} \sum_{s=1}^{k} \frac{d^{k-r}}{d t^{k-r}} Q_{k s}(t) \nabla^{2 m} f_{k-s} \\
& =\left(\nabla^{2 m}\right)^{-1} \sum_{s=1}^{k} Q_{r s}(t) \nabla^{2 m} f_{k-s} .
\end{aligned}
$$

The last formula proves that

$$
\frac{d^{k-r}}{d t^{k-r}} u \in w^{2 m}\left(E_{n}\right),
$$

$r=1,2, \ldots, k$, and $t \in(0,1)$.

Using (2.4) and (2.11) one gets,

$$
\frac{d^{k} u}{d t^{k}} \in w^{2 m}\left(E_{n}\right),
$$

for each $t \in(0,1)$.

In [5] we have proved that if $u, \frac{d u}{d t} \in w^{2 m}\left(E_{n}\right)$ and $\frac{d}{d t} D^{q} u \in L_{2}\left(E_{n}\right)$, $|q|=2 m, 4 m>n$, then the partial derivative $D_{t} u$ exists in the usual sense and that it is identical to the corresponding abstract derivative. Since these conditions are satisfied by $u$ in $(2.10)$, therefore the same conclusion applies. In a similar manner we can deduce also that the partial derivatives $D_{t}^{j} u, j=1,2, \ldots, k$ exist in the usual sense for each $t \in[0,1], x \in E_{n}$ and that they are identical to the corresponding abstract derivatives. 
Since $Q(0) G=G$, we have

$$
Q_{r s}(0)=\left\{\begin{array}{ll}
I & , r=s \\
0 & , r \neq s,
\end{array}\right\}
$$

therefore

$$
\nabla^{2 m} u(x, 0)=\sum_{s=1}^{k} Q_{k s}(0) \nabla^{2 m} f_{k-s}=\nabla^{2 m} f_{o}(x)
$$

The last formula leads to $u(x, 0)=f_{0}(x)$. In a similar manner we can prove that

$$
\mathrm{D}_{t}^{j} \mathrm{u}(\mathrm{x}, 0)=\mathrm{f}_{j}(\mathrm{x}), \mathrm{j}=0,1, \ldots, k,
$$

which complete the proof. (Compare [2]).

THEOREM 2. If the coefficients $\left(A_{q, j}(t),|q|=2 m, j=1,2, \ldots, k\right)$ commute with $D_{r}, r=1,2, \ldots, n$, then the solution of the problem (1.1), and (1.2) is given by the formula

$$
u=\sum_{s=1}^{k} Q_{k s}(t) f_{k-s}
$$

PROOF. For any $f \in W^{2 m}\left(E_{n}\right)$, we have $R^{q} \nabla^{2 m} f=\nabla^{2 m} R^{q} f$

Since the operators $\left(A_{q, j}(t)|q|=2 m, j=1, \ldots, k\right)$ commute with $D_{r}$, $r=1,2, \ldots, n$, it follows that the operators $\left(A_{q, j}(t)|q|=2 m, j=1, \ldots, k\right)$ commute with $\left(R^{q},|q|=2 m\right)$ and according to $(2.2)$ and (2.13) we can write

$$
\nabla^{2 m}\left[H_{0}(t) D_{t}^{k} u-\sum_{j=1}^{k} H_{j}(t) D_{t}^{k-j} u\right]=0
$$

The last equation leads immediately to

$$
D_{t}^{k} u=H_{0}^{-1} \sum_{j=1}^{k} H_{j}(t) D_{t}^{k-j} u .
$$

Applying similar steps to theorem (1), we obtain the required result.

COROLLARY. If the operators $\left(A_{q, j}(t),|q|=2 m, j=1, \ldots, k\right)$ commute with 
$D_{r}, r=1,2, \ldots, n$, then the Cauchy problem $(1.1),(1.2)$ is correctly

formulated.

PROOF. The proof of this important fact can be deduced immediately by using formula (2.12), (compare $[3],[4])$.

\section{REFERENCES}

[1] Calderon A.P., Zygmund A. Singular Integral Operators and Differential Equations 11, Amer, J. Math., 79, No. 3 (1957).

[2] Ehrenpreis L., Cauchy's Problem for Linear Differential Equations With Constant Coefficients 11, Proc. Nat. Acad. Sci. USA, 42, No. 9 (1956), 462-646.

[3] El-Borai M.M. On the Correct Formulation of the Cauchy Problem and the Mixed Problem for a Self-Conjugate Equation, Vectnik of Moscow Univ., 4. 1967.

[4] El-Borai M.M. On the Correct Formulation of the Cauchy Problem, Vectnik of Moscow University, 4, 1968.

[5] E1-Borai M.M. On the Initial Value Problem for Linear Partial Differential Equation With Variable Coefficients, Journal of Natural Science and Mathematics, Vol. XIV, No. 1 (1976).

[6] El-Bora1 M.M. Singular Integral Operators and Cauchy's Problem for Some Partial Differential Equations With Operator Coefficients, Transaction of the Science Centre, Alexandria University Vol. $2,1976$.

[7] Krein, C.G. Linear Differential Equations in Banach Space, Moscow (1967).

[8] Yosida K. Functional Analysis, Spreinger-Verlag, (1974). 


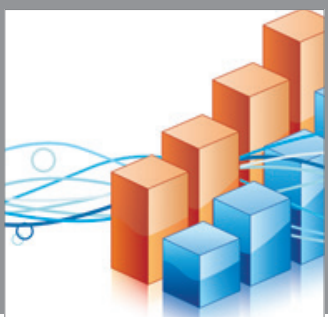

Advances in

Operations Research

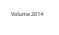

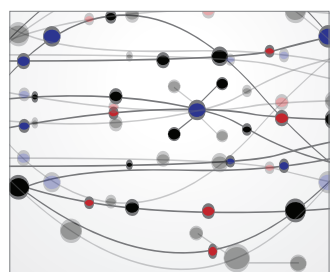

\section{The Scientific} World Journal
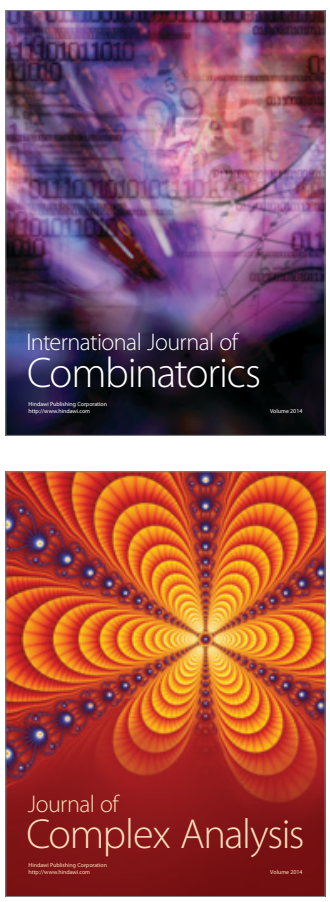

International Journal of

Mathematics and

Mathematical

Sciences
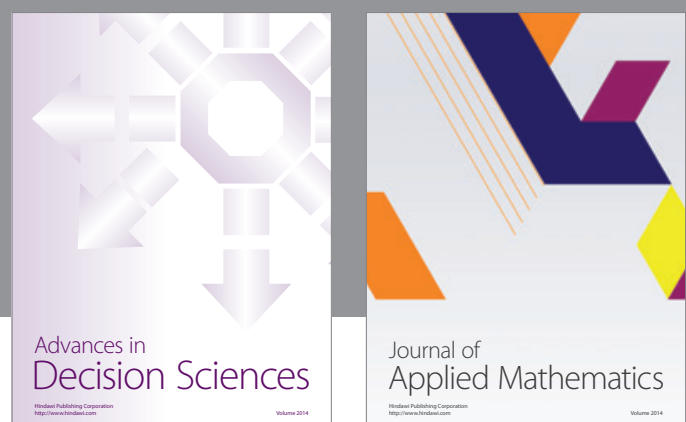

Journal of

Applied Mathematics
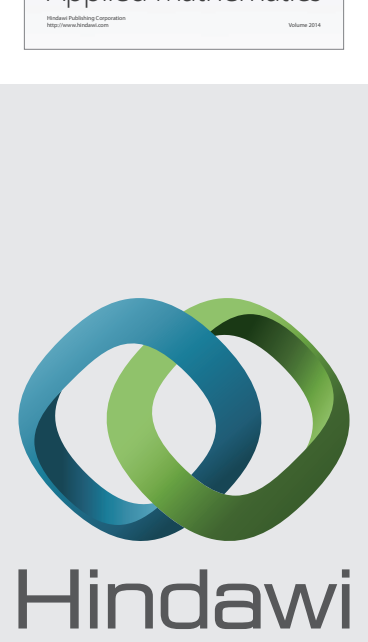

Submit your manuscripts at http://www.hindawi.com
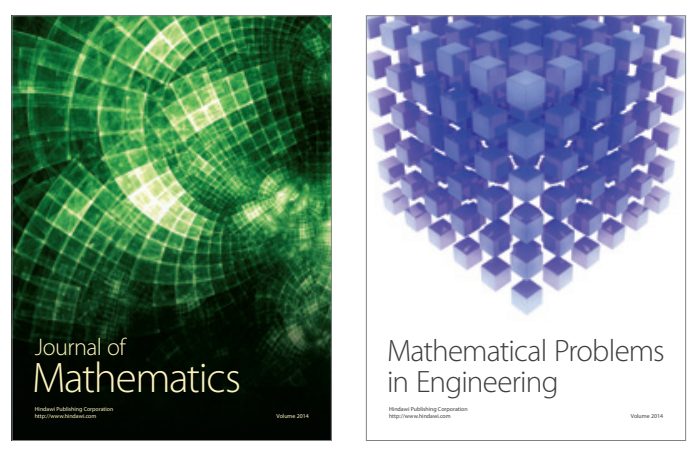

Mathematical Problems in Engineering
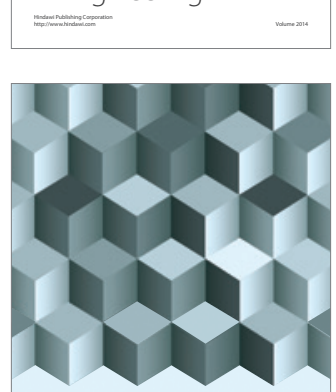

Journal of

Function Spaces
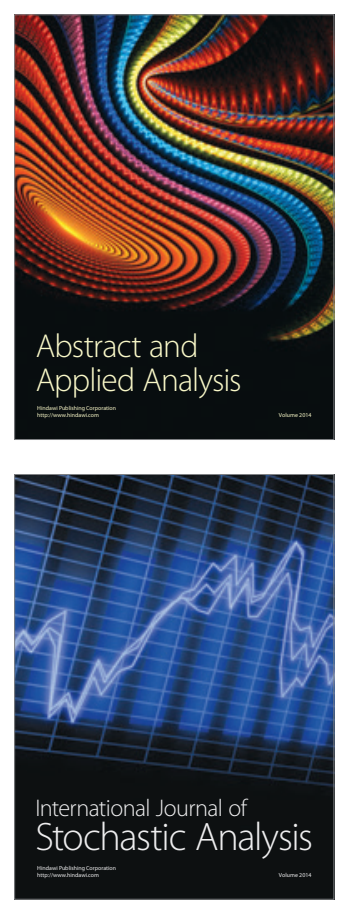

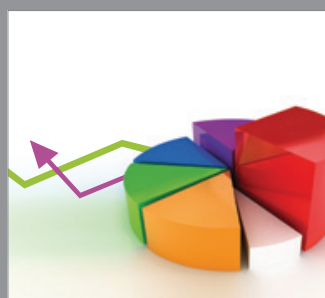

ournal of

Probability and Statistics

Promensencen
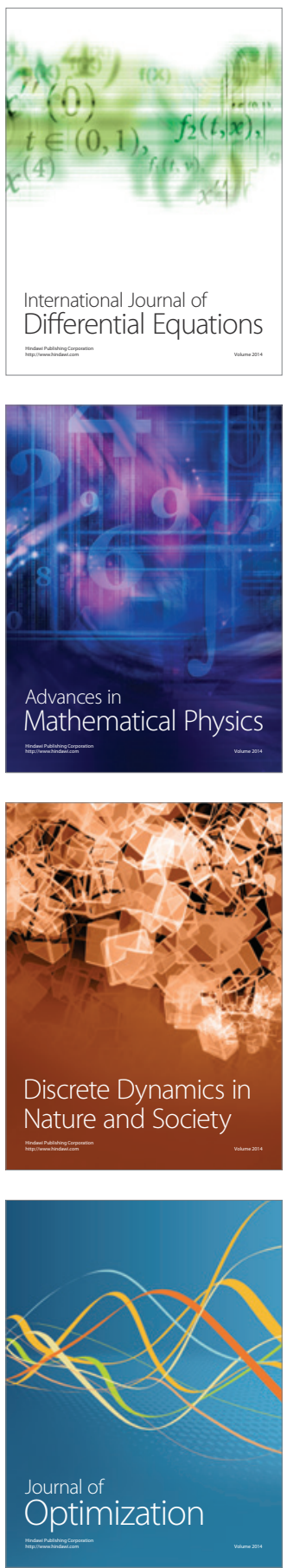